\title{
Performance Analysis of a Two Channel Optical WDM System using Binary and Duo Binary Modulation Formats
}

\author{
V. Khanaa ${ }^{1 *}$, Krishna Mohanta ${ }^{2}$ and T. Saravanan ${ }^{3}$ \\ 'Department of CSE, Sri Sai Ram Engg. College, Leo Nagar, Chennai 600 044, India; drvkannan62@yahoo.com \\ 2Department of ECE, Bharath University, Chennai-600073, India; krishnamohanta@gmail.com \\ ${ }^{3}$ Professor \& Head, Department of ETC, Bharath University, Chennai-600073, India; saravanan.etc@bharathuniv.ac.in
}

\begin{abstract}
The focus of this paper is to simulate a two channel optical WDM system for different bit rates. In this work, the comparison of both Binary and Duo binary modulation formats is carried out. Bit Error rate(BER) and Q factors are got improved with Duo binary modulation formats which will offer a significant performance benefits in digital systems.
\end{abstract}

Keywords: Dense Wavelength Division Multiplexing (DWDM), Continuous Wave (CW) Lasor, Bit Error Rate (BER), Erbium Doped Fiber Amplifier (EDFA), Q Factor, Eye Diagrams, Duo Binary.

\section{Introduction}

Nonlinear effects in optical fibers have become an area of academic research and of great importance in the optical fiber based systems. Optical duobinary coding is an effective method in high speed optical transmission systems to increase dispersion tolerance, to improve spectral efficiency and to reduce the sensitivity to non linear effects [6]. Optical duo-binary has attracted great attention in recent years. The term 'duo' means doubling the bit capacity of a straight binary system. The main advantages attributed to this modulation format are increased tolerance to the effects of chromatic dispersion. The fundamental idea of duo binary modulation (electrical or optical) that were first described by Lender in 1964 is to deliberately introduce Inter Symbol Interference (ISI) by overlapping data from adjacent bits [5]. This correlation between successive bits in a binary signal leads the signal spectrum to be more concentrated around the optical carrier. This is accomplished by adding a data sequence to a 1-bit delayed version of itself, which can be obtained by passing the binary signal through the delay-and-add filter [3]. Binary signal is a two level signal but duo binary is a three level signal. Binary has information capacity 1 , but duobinary has that of 2 also speed of duobinary is twice than that of Binary. For duobinary system, degree of complexity and amount of circuitry required are low.

\section{WDM Systems}

In fiber-optic communications, WDM is a technology which multiplexes multiple optical carrier signals on a single optical fiber by using different wavelengths (colours) of laser light to carry different signals. The term wavelengthdivision multiplexing is commonly applied to an optical carrier (described by its wavelength), whereas frequencydivision multiplexing typically applies to a radio carrier (described by frequency). However, since wavelength and frequency are inversely proportional, and since radio and light are both forms of electromagnetic radiation, the two terms are equivalent. Most WDM systems operate on single mode fiber optical cables, which have a core diameter of $9 \mu \mathrm{m}$. WDM systems are divided in different wavelength

*Corresponding author:

V. Khanaa (drvkannan62@yahoo.com) 


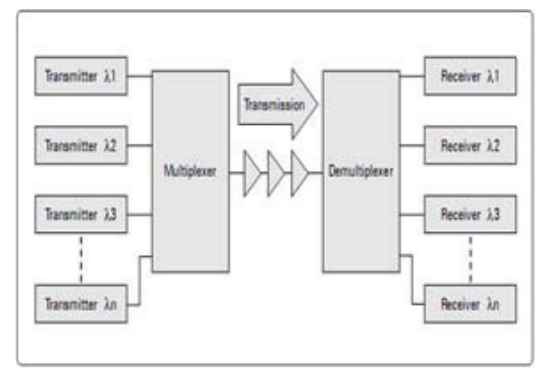

Figure 1. General WDM System.

patterns, conventional or coarse and dense WDM [4]. Conventional WDM systems provide up to 16 channels in the 3rd transmission window (C-band) of silica fibers around $1550 \mathrm{~nm}$. DWDM uses the same transmission window but with denser channel spacing.

Conventional WDM systems provide up to 16 channels in the 3rd transmission window (C-band) of silica fibers around $1550 \mathrm{~nm}$. DWDM uses the same transmission window but with denser channel spacing.

Very high-capacity, long-haul optical communication systems are made possible by the extremely wide bandwidth of optical fibers, which is best exploited by wavelength division multiplexing (WDM). WDM is the key technology of optical networking. Since wavelength and frequency are closely related to each other, this form of multiplexing is often called Frequency Division Multiplexing (FDM). Each WDM fiber has a certain bandwidth, the range of frequencies it can carry. One advantage of WDM is that every user can transmit information at the highest rate possible all the time. WDM does not transfer rates in proportion to the number of users on the line [2]. Another large benefit to WDM is that it increases the amount of information that can be transferred without significant loss of signal integrity. Figure 1 shows a general WDM system.

In the transmitter section a number of transmitters are provided which corresponds to different component wavelengths. Amplified signal is then fed into a DeMultiplexer (DMUX) for splitting it into different component wavelengths. At the receiving section a number of receivers are also provided for proper reception. Total transmission capacity can be enhanced by increasing. Total transmission capacity can be enhanced by increasing the number of WDM channels. Channel spacing and core effective area the main factors to be considered while designing a WDM optical communication link. WDM key features include capacity upgrade and transparency. Capacity upgrade means WDM increases the capacity of a fiber network.
Transparency means in WDM, each optical channel can carry any type of information (analog or digital) in any transmission formats simultaneously over the same fiber.

\section{Simulation Setup}

For high bit rate, long-haul communications supported by optical fiber, high quality light sources serving as transmitters are essential. Lasers operating in continuous wave (CW) mode provides a beam of light to an electro- optical modulator that is controlled to switch the light $\mathrm{ON}$ and OFF at the desired bit rate.

Two alternate ways to generate a modulated optical signal namely direct modulation and external modulation. In direct modulation, the laser is completely $\mathrm{ON}$ at all times and so called CW laser [3]. External modulation can produce higher quality optical pulses permitting extended reach and higher bit rates. Both direct and external modulation can produce NRZ or RZ modulated optical signals.

\section{Simulation Block Diagrams}

This work includes the simulation of a two channel WDM optical communication system in order to compare the BER and Q factor of both Binary and Duobinary modulation schemes. For the simulation purpose, here used optical software "OPSIM". Here we used Optsim 5.3. An optical fiber of $100 \mathrm{~km}$ long is used \& two CW semiconductor lasers externally modulated by Duo-binary \& binary modulation format like NRZ Rectangular.

\subsection{Using Binary Modulation Scheme}

In Binary modulation scheme, there are only two possible states- an ON state and an OFF state. Here ON state corresponds to logic ONE and OFF state corresponds to logic ZERO. Two $10 \mathrm{Gbps}$ data sources and CW Lasers are used. Figure 2 shows the block diagram of 2 channel optical WDM system employing Binary modulation scheme. Signals coming from the two channels are modulated by using optical modulators. These modulated signals are then combined in an optical combiner.

Combined signal is then fed to an optical amplifier and in this work here used an Erbium Doped Fiber Amplifier (EDFA). Amplified signal is then passed through a $100 \mathrm{~km}$ single mode fiber. At the receiving section a PIN photodiode is employed in order to convert optical signal into electrical form. Bessel electrical filter is also used for proper filtering. 


\subsection{Using Duobinary Modulation Scheme}

In Duo binary modulation scheme, there are two ON states and one OFF state. In both the ON states, the light is ON continuously with equal intensity but each ON state is ' $\mathrm{x} \pi$ ' radians out of phase [3]. Duo binary modulation format is obtained by driving an external dual arm Mach-Zhender modulator with opposite phase signal.

A Dual arm Mach-Zehnder modulator is based on Mach-Zhender Interferometer (MZI) and is fabricated from $\mathrm{LiNbO}_{3}[1]$. Two $10 \mathrm{Gbps}$ data sources and CW Lasers are used. An EDFA is used to amplify the signal coming from the optical combiner. Amplified signal is then transmitted through a $100 \mathrm{~km}$ standard single mode fiber. Figure 3 shows the block diagram 2 channel optical WDM using Duobinary modulation scheme.

While traveling through the fiber, the signal may be distorted due to dispersion or FWM. So in order to boost this signal an EDFA is again placed before detection. After amplification signal enters an optical splitter, which performs the function of an optical DMUX. A PIN photodiode is placed at the receiver section for optical to electrical conversion.

A Duo binary transmitter mainly consists of two NRZ rectangular drivers, Bessel electrical filters and a NOT gate. Here the NOT gate is used to invert the signal. NRZ

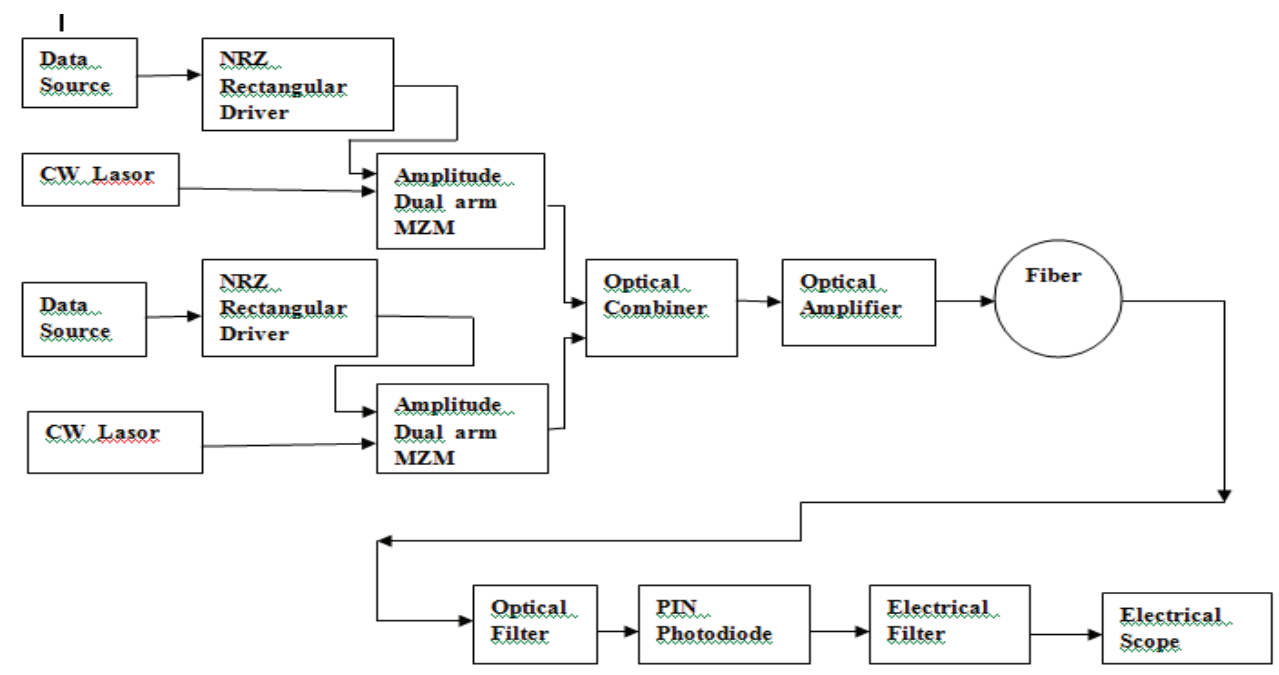

Figure 2. Block diagram of Duo binary modulation scheme for 2 channel WDM system.

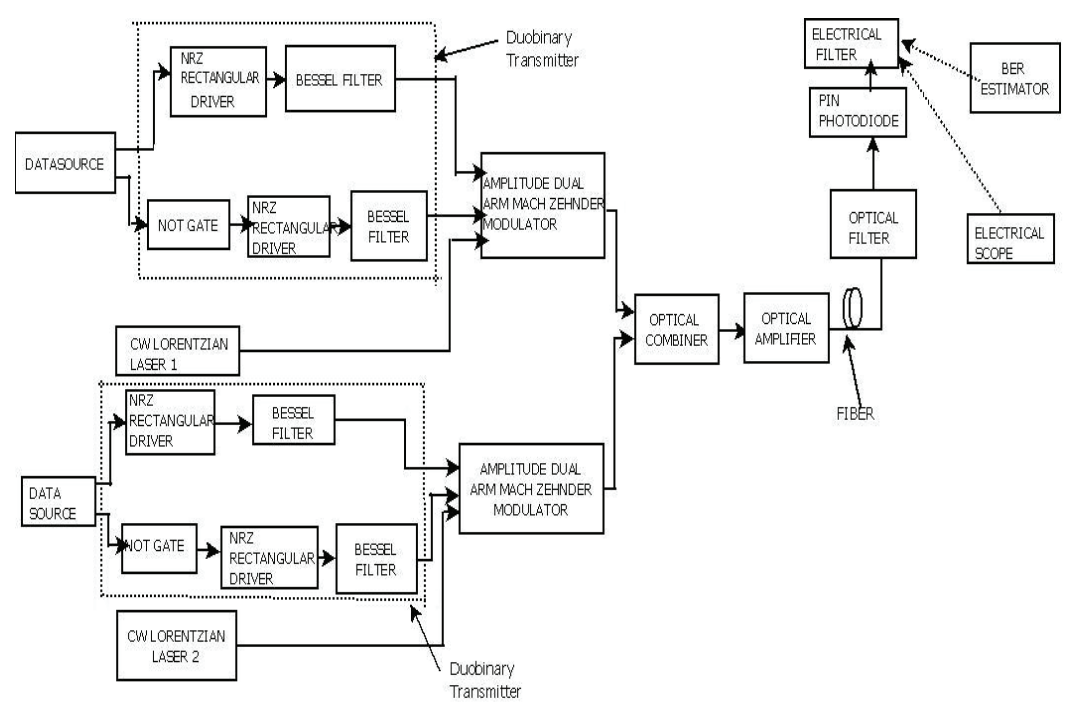

Figure 3. Block diagram of Duo binary modulation scheme for 2 channel WDM system. 
rectangular driver is the modulator drive [1]. Bessel electrical filter is a type of linear filer which preserves he wave shape. Bessel filter is also called Bessel-Thompson filter and it has maximally flat group delay.

\section{Simulation Schematics}

Optsim software was developed by RSoft Design Group. Optsim is an advanced optical communication system simulation package designed for professional engineering and cutting-edge research of WDM, DWDM, TDM, CATV, optical LAN, parallel optical bus, and other emerging optical systems in telecom, datacom, and other applications. It can be used to design optical communication systems and simulate them to determine their performance given various component parameters.

Optsim layouts of a two channel WDM optical communication system using both Binary and Duobinary modulation schemes are shown below. This simulation work is done in the Sample mode approach. In the sample mode simulation approach a component model will pass new sample data to another component model at each time step in the simulation.

\subsection{Optsim Layout of Binary Modulation}

Figure 4 shows the Optsim layout of Binary modulation format.

CW laser1 have central emission frequency 193.41449 $\mathrm{THz}(1550 \mathrm{~nm})$, optical power $3 \mathrm{dBm} \& \mathrm{FWHM}$ line width $10 \mathrm{MHz}$. CW Laser 2 have central emission frequency $193.45817 \mathrm{THz}$ (1549.65nm) [3]. Optical spectrum can be viewed through OSA and eye diagrams through electrical scopes.

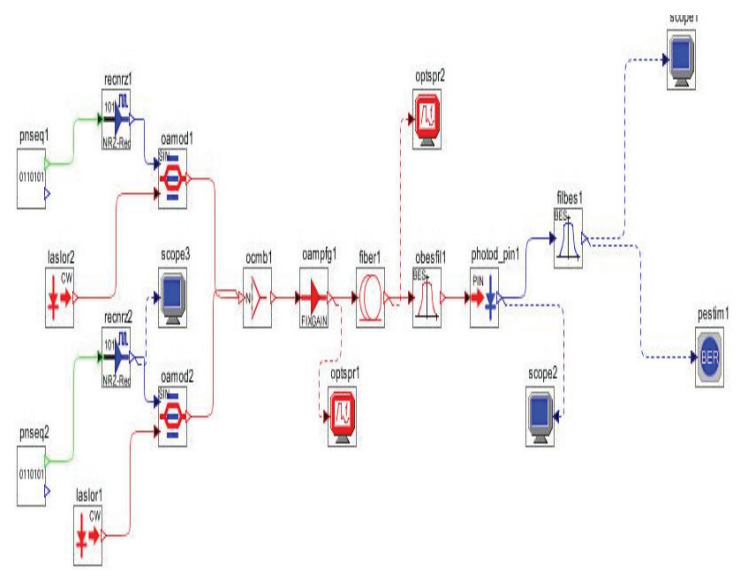

Figure 4. Binary modulation schematic.

\subsection{Optsim Layout of Duo Binary Modulation}

Figure 5 shows the Optsim layout of Duobinary modulation format.

Data source generate data of various bit rates in Gbps. The duo binary encoder used consists of a one-bit delay line. The output of the delay line is added to the original signal to generate a zero mean, three-level signal.

Here external modulation is carried out by amplitude dual arm Mach Zehnder modulator (MZM). The Mach Zehnder modulator has maximum transmissivity offset voltage of $0.5 \mathrm{~V} \&$ extinction ratio of $20 \mathrm{~dB}$. Same external modulator is used for both duo binary \& binary signal with same parameter as mentioned above. Duo binary modulation format is obtained by driving an external dual arm modulator with opposite phase signal.

Optical spectrum can be viewed through OSA and eye diagram through electrical scope. From the eye diagrams, BER, Q values etc can be determined. Optical spectrum gives both frequency and peak power of the optical signals.

\section{Simulation Results and Discussions}

Simulation results can be obtained through the various measurement components like Optical Spectrum Analyzer (OSA) and Electrical scope which provides Eye diagrams, BER Estimator, Electrical probes etc. The spectrum after propagation through the fiber is viewed on an OSA. At receiver side, electrical scope is connected to electrical filter output to view eye diagram, to calculate BER, Q factor. Eye diagrams can be used o effectively analyze the performance

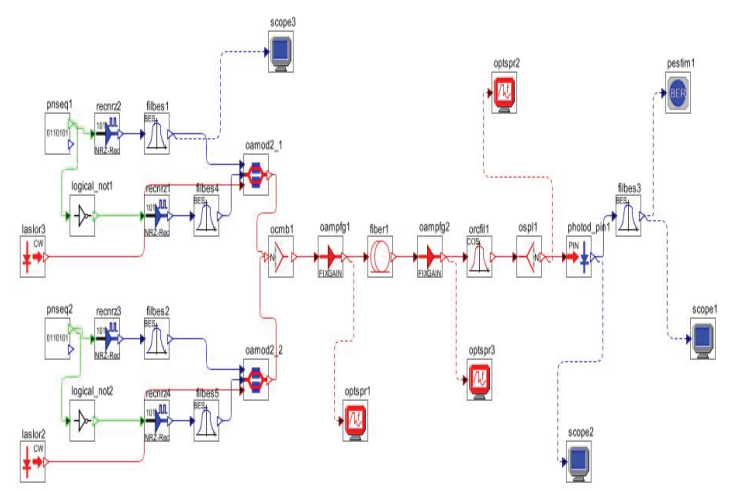

Figure 5. Duo binary modulation schematic. 
of an optical system. If the eye opening is very wide here is no crosstalk. Eye height is an indicator of noise. Signal width at the centre of an eye diagram represents a measure of timing jitter.

\subsection{Results from Binary Modulation}

\subsubsection{Optical Spectra}

When we analyze the above spectra, two highest peaks are appeared which corresponds to two channels of different wavelengths used in this work. At the fiber output, the highest peak power is reduced after travelling through a $100 \mathrm{~km}$ long optical fiber cable due to some linear and non linear effects.

\subsubsection{Eye Diagrams}

Eye diagrams obtained for different bit rates in Duobinary modulation scheme are shown in Figure 7. These eye diagrams are obtained at the receiving section after PIN photodiode (before and after filtering).

Eye opening becomes wider while reducing the bit rates. For a bit rate of $2.5 \mathrm{Gbps}$, a clear eye opening is obtained and $\mathrm{BER}$ is reduced which results in better $\mathrm{Q}$ facor. There is no eye opening is present when bit rate is $20 \mathrm{Gbps}$ and eye diagram is completely distorted.

BER and Q factor values for different bit rates are summarized (Table 1, Figure 8).

As bit rate is increased, BER is also increased which results in poor $\mathrm{Q}$ factor.
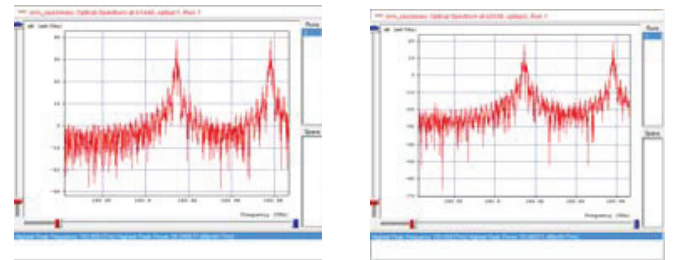

Figure 6. Optical spectra obtained from (a) fiber input and (b) fiber ouput in Binary modulation scheme.

Table 1. Results from Eye diagrams of Binary modulation

\begin{tabular}{ccclc}
\hline $\begin{array}{l}\text { Bit Rate } \\
\text { (Gbps) }\end{array}$ & \multicolumn{2}{c}{ Scope 1 } & \multicolumn{2}{c}{ Scope 2 } \\
\cline { 2 - 5 } & \multicolumn{1}{c}{ BER } & $\begin{array}{c}\text { Q Value } \\
(\mathrm{dB})\end{array}$ & \multicolumn{1}{c}{ BER } & $\begin{array}{c}\text { Q Value } \\
(\mathrm{dB})\end{array}$ \\
\hline 20 & 0.0199879 & 6.536551 & 0.0227501 & 6.020600 \\
10 & 0.00125111 & 9.632181 & 0.00480456 & 8.299471 \\
7.5 & 0.0021753 & 10.038652 & 0.0036921 & 9.573924 \\
5 & 0.00043307 & 10.452398 & 0.00113719 & 9.737243 \\
2.5 & 0.000289204 & 11.020758 & 0.000624929 & 10.177290 \\
\hline
\end{tabular}
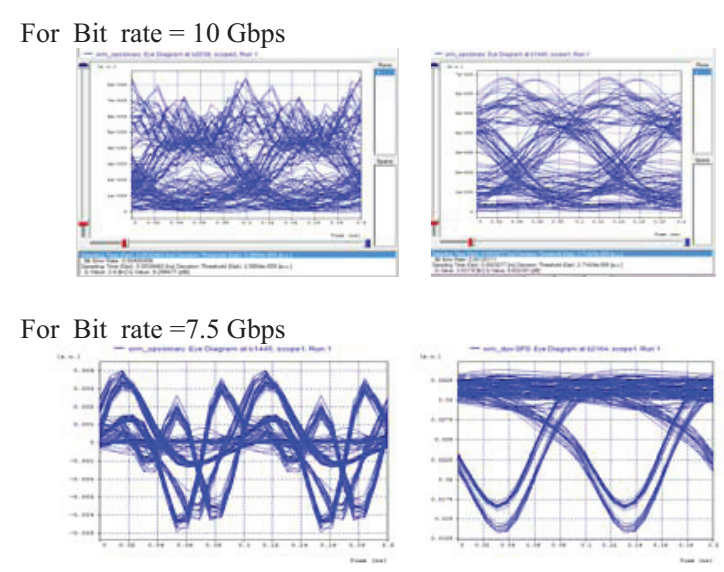

For Bit rate $=5 \mathrm{Gbps}$
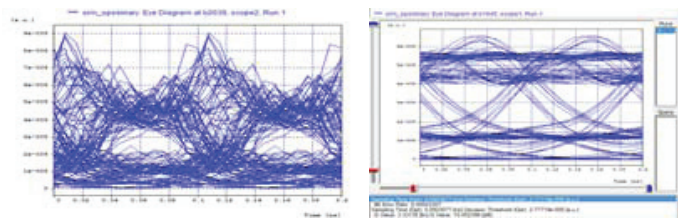

For Bit rate $=2.5 \mathrm{Gbps}$
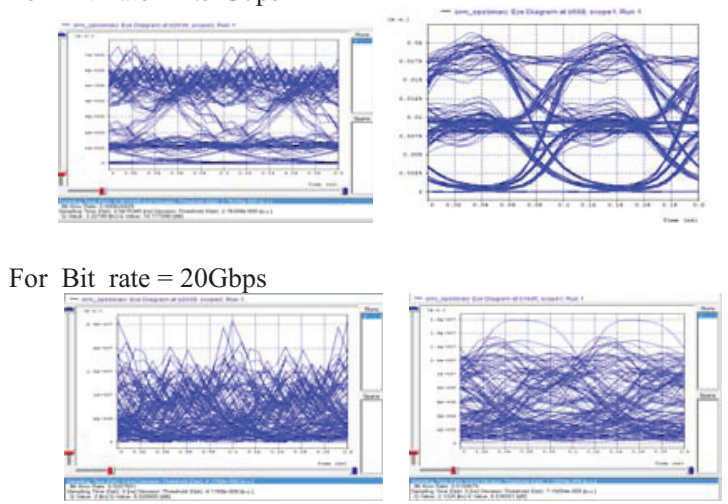

Figure 7. Eye diagrams for different bit rates obtained in Binary modulation.
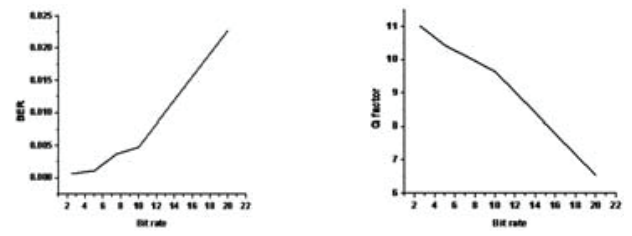

Figure 8. Variation of BER and $\mathrm{Q}$ factor with Bit rate of Binary modulation.

\subsection{Results from Duo Binary Modulation}

\subsubsection{Optical Spectra}

Figure 9 represents the optical spectra obtained in Duo Binary modulation. 

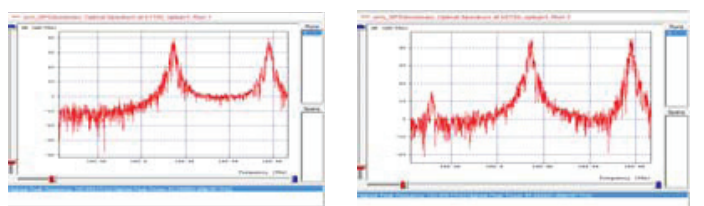

Figure 9. Optical spectra obtained from (a) fiber input and (b) fiber ouput in Duo Binary modulation scheme.

When we analyze the above spectra, an additional optical side band (small peak) along with two highest peaks is appeared which corresponds to the Four Wave Mixing (FWM) product. At the fiber output, the highest peak power is reduced after travelling through a $100 \mathrm{~km}$ long optical fiber cable due to FWM effects. But it is increased a little after amplification by EDFA.

\subsubsection{Eye Diagrams}

Eye diagrams obtained for different bit rates in Duobinary modulation scheme are shown (Figure 10). These eye diagrams are obtained at the receiving section after PIN photodiode (before and after filtering).

Eye opening becomes wider for lower bit rates. For a bit rate of $2.5 \mathrm{Gbps}$, a clear eye opening is obtained and BER is reduced which results in better $\mathrm{Q}$ facor. There is no eye opening is present when bit rate is $20 \mathrm{Gbps}$ and at his rate, eye diagram is completely distorted. While comparing the eye diagrams, it is revealed that eye diagrams obtained in Duo binary modulation scheme are better than that obtained in Binary modulation.

BER and Q factor values for different bit rates are summarized in Table 2, Figure 11.

As bit rate is increased, BER is also increased which results in poor $\mathrm{Q}$ factor.

\subsubsection{Binary vs. Duobinary}

Figure 12 shows the comparison of Q factor vs. Bit rate in Binary and Duo binary modulation formats for different bit rates.

From the above graph, it is concluded that Q factor of Duo binary modulation format is better than Binary modulation for various bit rates. Better $\mathrm{Q}$ factors are obtained at a bit rate of $2.5 \mathrm{Gbps}$ for both Binary and Duobinary modulation schemes. For 5Gbps, obtained Q factors are $11 \mathrm{~dB}$ and $19 \mathrm{~dB}$ in binary and Duo binary modulation formats respectively.

\section{Conclusion}

In this work, the simulation of a 2 channel WDM optical communication system for different modulation formats

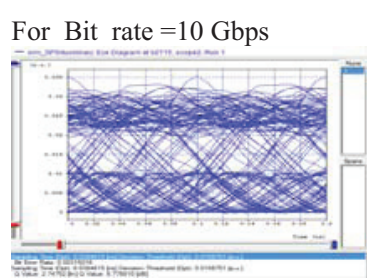

For Bit rate $=7.5 \mathrm{Gbps}$
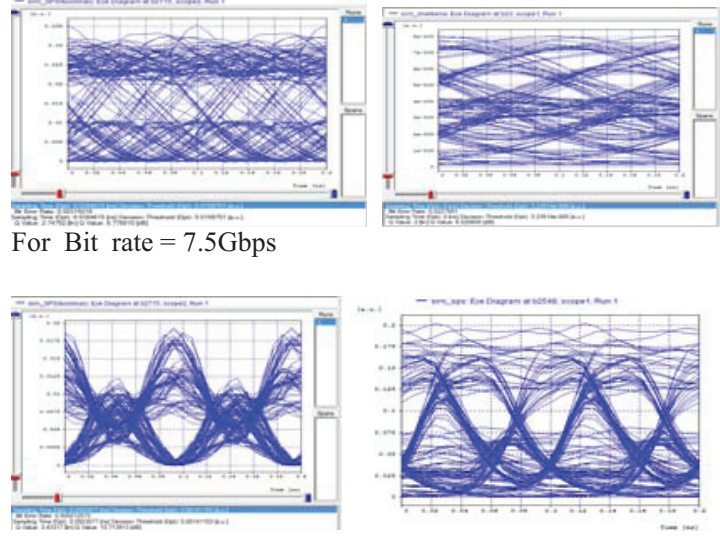

For Bit rate $=5 \mathrm{Gbps}$
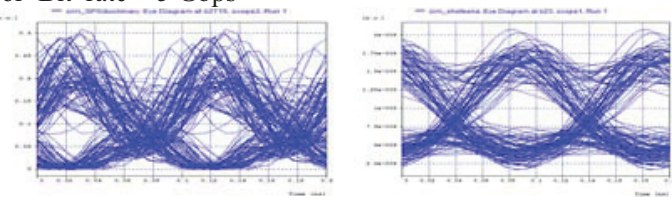

For Bit rate $=2.5 \mathrm{Gbps}$
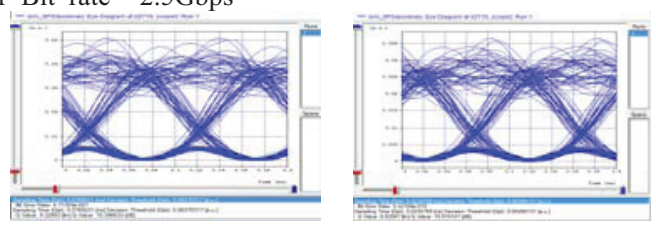

For Bit Rate $=20 \mathrm{Gbps}$
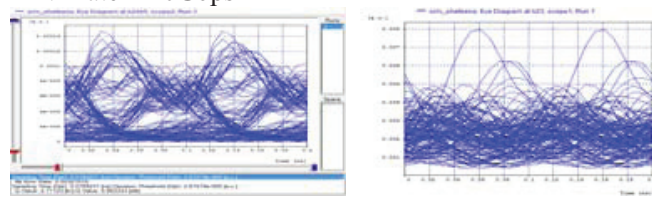

Figure10. Eye diagrams for different bit rates obtained in Duo Binary modulation.

Table 2. Results from Eye diagrams of Binary modulation

\begin{tabular}{ccccc}
\hline $\begin{array}{c}\text { BitRate } \\
\text { (Gbps) }\end{array}$ & \multicolumn{2}{c}{ Scope 1 } & \multicolumn{2}{c}{ Scope 2 } \\
\cline { 2 - 5 } & \multicolumn{1}{c}{ BER } & $\begin{array}{c}\text { Q Value } \\
(\mathrm{dB})\end{array}$ & \multicolumn{1}{c}{ BER } & $\begin{array}{c}\text { Q Value } \\
(\mathrm{dB})\end{array}$ \\
\hline 20 & 0.00359216 & 6.020600 & 0.00307019 & 8.663333 \\
10 & 0.0227501 & 6.052317 & 0.00315016 & 8.778810 \\
7.5 & 0.0053869 & 9.362857 & 0.00021257 & 10.713913 \\
5 & 0.0007256 & 12.0053683 & 0.0019379 & 11.020758 \\
2.5 & $4.7135 \mathrm{e}-021$ & 19.396633 & $5.2218 \mathrm{e}-019$ & 18.915147 \\
\hline
\end{tabular}

is presented in order to analyze the variations in BER and Q factor with different bit rates. Here used both Binary 

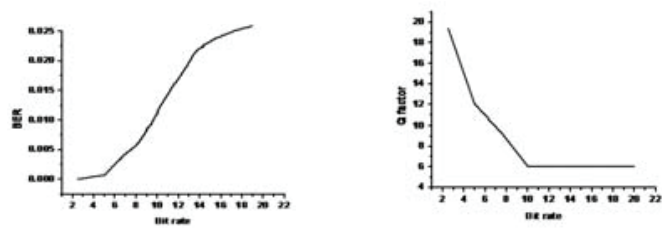

Figure 11. Variation of BER and Q factor with Bit rate of Duo Binary modulation.

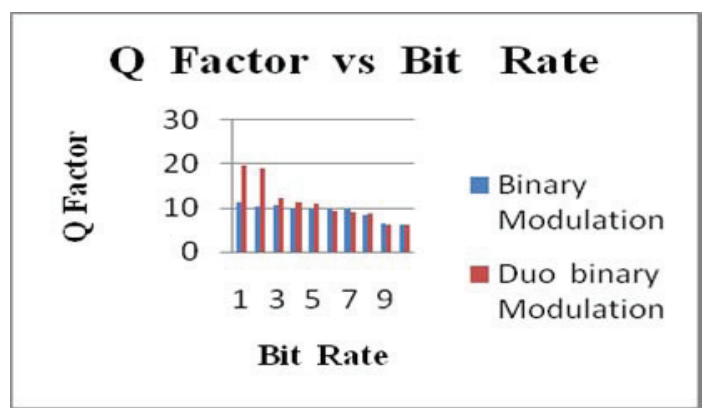

Figure 12. Comparison of $\mathrm{Q}$ factor in Binary and Duo binary modulation schemes.

and Duo binary modulation formats for this purpose. From the obtained results it is concluded that BER is got improved with duo binary modulation format than a binary scheme for different values of bit rates which will offer a significant performance benefit in digital systems. For the bit rates of $5 \mathrm{Gbps}$ and $2.5 \mathrm{Gbps}$, Duo binary modulation scheme gives highest $\mathrm{Q}$ factor than Binary modulation scheme

\section{References}

1. Tawade L, and Deokate B (2011). The reduction of FWM effects using duobinary modulation in a two-channel D-WDM system, International Journal of Distributed and Parallel Systems, vol 2, No. 6, 303-307.

2. Rahman M Z, and Islam M S (2011). Effect of chromatic dispersion on four-wave mixing in WDM optical transmission system, International Journal on Internet and Distributed Computing Systems, vol 1, No. 2.

3. Tawade L, Jagdale $S$ et al. (2010). Investigation of FWM effect on BER in WDM optical communication system with binary and duobinary modulation format, International Journal of Distributed and Parallel Systems, vol 1, No. 2, 109-116.

4. ElRazak B K A, and Saleh M B (2011). Duobinary Modulation format and unequal channel spacing integration to suppress four-wave mixing crosstalk in WDM systems, 2011 Saudi International Electronics, Communications and Photonics Conference (SIECPC).

5. Lender A (1963). The duobinary technique for high speed data transmission, Transactions of the American Institute of Electrical Engineers, Part I: Communication and Electronics, vol 82(2), 214-218.

6. Kaiser W, Wuth $\mathrm{T}$ et al. (2001). Reduced complexity optical duobinary $10 \mathrm{~Gb} / \mathrm{s}$ transmitter setup resulting in an increased transmission distance, IEEE Photonics Technology Letters, vol 13(8).

7. Shibata N, Nosu K et al. (1999). Transmission limitations due to fiber nonlinearities in optical FDM systems, IEEE Journal on Selected Areas in Communications, vol 8(6), 1068-1077. 